\title{
The Expression of VEGFR2 and VEGFR3 in Early Gastric Cancer and Its Clinical Significance
}

Xiu-Feng $\mathrm{Li}^{1,2^{*}}$, Yun-Xiang Zhang ${ }^{2}$, Ting-Guo Zhang ${ }^{1}$ and Min Yang ${ }^{3}$

${ }^{1}$ Shandong University School of Medicine of China, Lixia, Jinan, China

${ }^{2}$ The People's Hospital of Wei Fang City, Shan Dong Province, China

${ }^{3}$ Pathology Department, Qi Lu Hospital, Shandong University, Lixia, Jinan, China

*Corresponding author: Xiu-Feng Li, Shandong University School of Medicine of China, Lixia, Jinan, China, Tel: +86 53188395114 ; E-mail: lixiufeng.82@163.com Received date: April 25, 2018; Accepted date: April 27, 2018; Published date: May 05, 2018

Copyright: (c) $2018 \mathrm{Li} \mathrm{XF}$, et al. This is an open-access article distributed under the terms of the Creative Commons Attribution License, which permits unrestricted use, distribution, and reproduction in any medium, provided the original author and source are credited.

\section{Abstract}

Background: To explore the expression and its clinical significance of vascular endothelial growth factor receptor II and III (VEGFR2 and VEGFR3) in early gastric cancer.

Methods: Immunohistochemical method is applied to detect 120 cases of early gastric cancer and normal gastric tissue of its cut edge in the expression of VEGFR2 and VEGFR3.

Results: The positive expression rates of VEGFR2 and VEGFR3 in early gastric cancer are $75 \%$ and $60 \%$, which are higher than in normal gastric tissues of cut edge $(22.5 \%, 12.5 \%)$, respectively. The expression of VEGFR2 and VEGFR3 is associated with depth of invasion, lymph node metastasis $(P<0.05)$, regardless of patient's gender, age, tumor size, gross classification, differentiation $(P>0.05)$.

Conclusion: VEGFR2 and VEGFR3 play important roles in occurrence and development of early gastric cancer, which potentially provides a basis for targeted molecular therapy of early gastric cancer.

Keywords: Gastric cancer; Tumor; Immunohistochemical; Materials and Methods

Intramucosal; Lymphatic invasion
Abbreviations
VEGFR2: Vascular Endothelial Growth Factor Receptor II; VEGFR3: Vascular Endothelial Growth Factor Receptor III; EGC: Early-Gastric Cancer.

\section{Background}
Gastric cancer is one of the most common malignant tumors, which is difficult to cure. Vascular endothelial growth factor receptor 2 is influenced by tyrosine kinase. Some studies have shown that VEGFR2 is closely related to the formation of tumor blood vessels, tumor staging, metastasis, and prognosis and treatment effect. Vascular endothelial growth factor receptor 3 is lymphatic endothelial markers, and its expression is closely related to tumor lymph node metastasis. This study is to investigate both VEGFR2 and VEGFR3 expression in early gastric cancer by immunohistochemical method. The relationship between their expression and the tumor clinical and pathological features is analyzed. This study will provide a base in understanding the occurrence and development of early gastric cancer and targeted molecular therapy.

\section{General information}

Selection of Qi Lu hospital of Shan Dong university from 2013 to 2014 surgical resection of early gastric carcinoma specimens of 120 cases, including 69 cases of male and 51 cases of female, which average age is 54 . The conventional pathology of the 120 cases is all reviewed by two highly qualified pathologists. In strict accordance with the 2006 edition of the world health organization of tumor classification and diagnostic criteria of series standard [1], 48 cases are well and moderately differentiated adenocarcinoma, 72 cases are poorly differentiated adenocarcinoma (including signet ring cell carcinoma, mucous adenocarcinoma); 39 cases are intramucosal carcinoma, 81 cases are with submucosal invasion; 20 cases are with lymph node metastasis, 100 cases are without lymph node metastasis. 80 cases of normal gastric tissues were collected as control from the 120 cases of early gastric cancer cut edge. In order to make clinical data integrity, preoperative patients in these trials were not accepted by radiotherapy and chemotherapy.

\section{Reagent and method}

Rabbit polyclonal antibody against human VEGFR2 (ZA-0287), rat monoclonal antibody against human VEGFR3 (ZM-0277) and biotinStreptomyces antibiotic immunohistochemical kit (SP-9001) and DAB chromogenic agent (ZLI-9018) were bought from Beijing Chinese fir golden bridge biotechnology company. Specific steps were acted in strict accordance with the instructions, each cancer tissues and normal tissues of cut edge wax block (including $4 \mathrm{~m}$ serial section), 
Citation: Li XF, Zhang YX, Zhang TG, Yang M (2018) The Expression of VEGFR2 and VEGFR3 in Early Gastric Cancer and Its Clinical Significance. J Bioprocess Biotech 8: 325. doi:10.4172/2155-9821.1000325

Page 2 of 4

respectively for Streptomyces avidin peroxidase connection method of staining (SP) method, meanwhile $0.1 \mathrm{~mol} / \mathrm{L}$ phosphate buffer instead of one as negative control.

\section{The result interpretation}

Stained slides were examined under light microscope on the premise of exclusion of nonspecific staining, resulting in the cytoplasm material as positive cells. Based on stain levels, cases are classified as: positive cells $<10 \%$ as negative $(-), 10 \% \sim 25 \%$ positive cells as weakly positive $(+)$, positive cells in $26 \% \sim 50 \%$ as positive $(++)$, positive cells $>50 \%$ as strongly positive $(+++)$. To simplify the calculation, positive expression is expressed as $+\sim+++$.

\section{Statistics processing}

Application of SPSS 11.0 statistical software, count data chi-square test and Fisher's exact probability method, with $\mathrm{P}<0.05$ for the difference was statistically significant.

\section{Results}

\section{The expression of VEGFR2 and VEGFR3 in early gastric} cancer

Both VEGFR2 and VEGFR3 positive expression in early gastric cancer tissue are yellow particles, especially in tumor cell cytoplasm tumor (Figure 1).

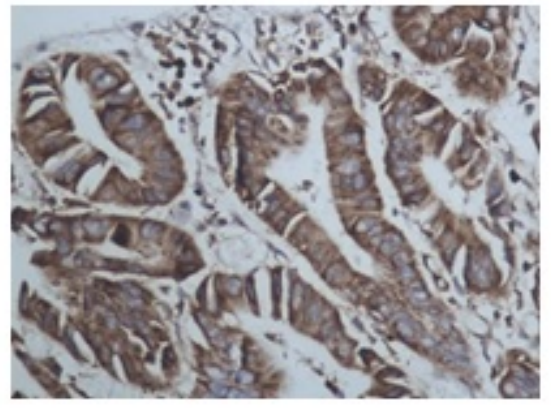

VEGFR2 expression

\section{(400 tim es)}

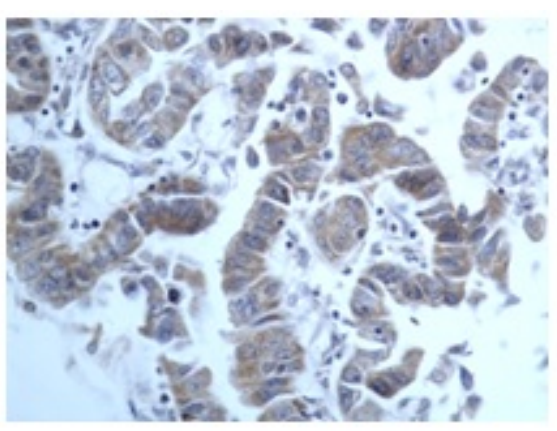

VEGFR3 expression (400 tim es)

Figure 1: Expression of VEGFR2 and VEGFR3.

There is a significant difference $(\mathrm{P}<0.05)$ of expression positive rate in early gastric cancer tissues and normal gastric mucous tissues, shown in Table 1.

\begin{tabular}{|l|l|l|l|l|l|l|l|}
\hline \multicolumn{2}{|l|}{ VEGFR2 } & + & \multicolumn{3}{l|}{ VEGFR3 } & & \\
\hline Cases & + & - & P & + & - & P \\
\hline $\begin{array}{l}\text { Early gastric } \\
\text { cancer }\end{array}$ & 120 & 90 & 30 & & 72 & 48 & \\
\hline Normal gastric & 80 & 18 & 62 & $<0.05$ & 10 & 70 & $<0.05$ \\
\hline
\end{tabular}

Table 1: The expression of VEGFR2 and VEGFR3 in early gastric cancer and normal gastric tissues.

In early gastric cancer tissues, the expression positive rate of VEGFR2 and VEGFR3 are 75\% (90/120) and 60\% (72/120), respectively. In normal gastric mucous tissues, the expression positive rate of VEGFR2 and VEGFR3 are 22.5\% (18/80) and 12.5\% (10/80), respectively. It can be seen that the positive rate of VEGFR2 and VEGFR3 in sub mucosal carcinoma $(81.5 \%, 66.7 \%)$ are higher than the positive rate in mucosal carcinoma $(61.5 \%, 55 \%)$. In contrast to without lymph node metastasis, the positive rate of patients has a significant improvement with lymph node metastasis. For VEGFR2, the positive rate increases from $71 \%$ to $95 \%$. For VEGFR3, the value increases from $55 \%$ to $85 \%$. Also from Table 2.

\begin{tabular}{|l|l|l|l|l|l|l|l|l|}
\hline Clinical & & & & VEGFR2 & & & VEGFR3 & \\
\hline Parameters & & Case & Positive & Negative & P & Positive & Negative & P \\
\hline Sex & Male & 69 & 51 & 18 & & 43 & 26 & \\
\hline
\end{tabular}




\begin{tabular}{|c|c|c|c|c|c|c|c|c|}
\hline & Female & 51 & 39 & 22 & & 29 & 22 & \\
\hline \multirow{2}{*}{ Patient age } & 60 & 87 & 67 & 20 & & 48 & 39 & \\
\hline & $\geq 60$ & 33 & 23 & 10 & & 24 & 9 & \\
\hline \multirow{2}{*}{ Tumor size } & $2 \mathrm{~cm}$ & 39 & 30 & 9 & & 20 & 19 & \\
\hline & $\geq 2 \mathrm{~cm}$ & 81 & 60 & 21 & & 52 & 29 & \\
\hline \multicolumn{9}{|c|}{ Tumor differentiation } \\
\hline Low grade & & 48 & 39 & 9 & & 27 & 21 & \\
\hline High grade & & 72 & 51 & 21 & & 45 & 27 & \\
\hline \multicolumn{9}{|c|}{ Depth of invasion } \\
\hline Mucosal & & 39 & 24 & 15 & 0.05 & 18 & 21 & 0.05 \\
\hline Submucosal & & 81 & 66 & 15 & & 54 & 27 & \\
\hline \multicolumn{9}{|c|}{ Lymph node metastasis } \\
\hline Without & & 20 & 19 & 1 & 0.05 & 17 & 3 & 0.05 \\
\hline With & & 100 & 71 & 29 & & 55 & 45 & \\
\hline Gross feature & & 12 & 9 & 3 & & 9 & 3 & \\
\hline \multicolumn{9}{|l|}{ Uplift } \\
\hline Superficial & & 42 & 30 & 12 & & 27 & 15 & \\
\hline Depressed & & 66 & 51 & 15 & & 36 & 30 & \\
\hline
\end{tabular}

Table 2: VEGFR2 and VEGFR3 expression and its clinical pathological relationship in early gastric cancer.

It can be seen that sex, age, tumor size, gross classification, and tumor differentiation do not significantly affect VEGFR2 and VEGFR3 expression positive rates.

\section{Discussion}

In recent years, with the popularization of health knowledge and advance of gastroscopy and medical technology, the discovery rate of early gastric cancer has increased year by year. Referring to the depth of tumor invasion, early gastric cancer (EGC) has been confined to the mucosa or submucosa, which has nothing to do with the tumor size and lymph node status (with or without metastasis)

VEGFR2, mainly by adjusting the permeability of vascular endothelial cells, promotes cell mitosis and chemotaxis, the formation of new blood vessels and maintenance of endothelial cells survive. But it prevents endothelial cell apoptosis. The formation of new blood vessels is beneficial to tumor growth, and it can provide the channel of tumor metastasis. Studies have confirmed that VEGFR2 is related with the number of new blood vessels, lymph node metastasis, liver metastasis and the malignant transformation of gastric cancer. As demonstrated by this study, VEGFR2 expression in early gastric cancer tissues is significantly higher than in the gastric normal mucosa. This study also indicated that the expression of VEGFR2 is related to depth of invasion, lymph node metastasis, regardless of gender, age, tumor size, gross feature, and tumor differentiation. These results suggest that VEGFR2 plays a role in the occurrence and development of early gastric cancer, associated with tumor invasion and metastasis. In recent studies, Fuchs [2] have confirmed that VEGFR2 signaling pathway is an important drug targets in highly differentiated gastric cancers, and the VEGFR2 targeted therapy can significantly improve the prognosis of patients with gastric cancer. Therefore VEGFR2 may become the ideal target of early gastric cancer treatment.

It is firstly found that VEGFR3 is a lymphatic specific growth factor receptor. In normal adult tissue, VEGFR3 is mainly expressed in the lymphatic endothelial cells, and it is not expressed in vascular endothelial cell. Han [3] confirmed VEGF-C and VEGFR-3 expressions in gastric cancer are associated with lymph node metastasis. Choi [4] found VEGFR3 positive lymphatic density increased significantly in gastric cancer, which is related with the primary tumor size, lymphatic invasion, and lymph node metastasis. As a result of this study, VEGFR3 expression in early gastric cancer is significantly higher than in the gastric normal mucosa. The expression of VEGFR3 protein is related with depth of invasion, lymph node metastasis, regardless of gender, age, tumor size, gross feature, tumor differentiation. These finds suggest that VEGFR3 is involved in the occurrence and development of early gastric cancer, and is associated with the tumor invasion and metastasis.

Lymphatic channel is the important way of gastric cancer metastasis, and lymphatic invasion is one of the key factors influencing the prognosis of patients. The lymph node metastasis rate in early gastric cancer can reach $5.7 \%$ to $20 \%$, and patients without lymph node metastasis always have a better prognosis than patients with lymph node metastasis. VEGF-C/D is the first identified as specific lymphatic vessel growth factor, and it is a new lymphatic tumor related piping 
Citation: Li XF, Zhang YX, Zhang TG, Yang M (2018) The Expression of VEGFR2 and VEGFR3 in Early Gastric Cancer and Its Clinical Significance. J Bioprocess Biotech 8: 325. doi:10.4172/2155-9821.1000325

Page 4 of 4

formation. Also, VEGF-C/D is very important in the process of signal conditioning factors. VEGF-C/D and its receptor in tumor lymphatic growth play an important role and have become a focus of gastric cancer study. Recent research suggests VEGF-C/D and VEGFR-2 in certain conditions can also induce lymphatic generation [5]. VEGFR-3 is specifically combined with VEGF-C/VEGF-D in maintaining occurrence of the lymphatic system, the structure and function of the lymphatic vessels and lymphatic freshman. VEGF-C/D, after combining with VEGFR-3, causes downstream molecular Shc Grb2 phosphorylation by MARK signal and prompts lymphatic endothelial cell proliferation, migration, survival and formation of new lymphatic ducts, thus providing favorable conditions for tumor cell lymphatic invasion and tumor metastases. Studies about molecular treatment have shown that blocking VEGFR3 mediated signal transduction pathways can effectively inhibit tumor lymphatic generation, and inhibit the lymph node metastasis, which suggests that VEGFR3 could be effective molecular therapy targets [6]. As further illustrated by our study, VEGF-C/D and its receptor can be used as potential indicators for lymph node metastasis, and an important evaluation factors in estimating the prognosis of patients with gastric cancer. At the same time, comparing with VEGF-C/D and VEGFR2, VEGFR3 axis interference is expected to become a new breakthrough, and it provides a new path in the treatment of early gastric cancer with lymph node metastasis. So the measurement of VEGFR-2 and VEGFR-3 expression in patients with early gastric cancer should has a positive role in evaluating the prognosis and it is more important in the gastric cancer treatment.

\section{Conclusion}

VEGFR2 and VEGFR3 play important roles in occurrence and development of early gastric cancer, which potentially provides a basis for targeted molecular therapy of early gastric cancer.

\section{References}

1. Hamilton SR, Aaltonen LA (2006) The world health organization (WHO) tumor classification and diagnostic criteria of series: Digestive system tumor pathology and genetics. Beijing: People's Medical Publishing House, pp: 42-59.

2. Fuchs CS, Tomasek J, Yong CJ, Dumitru F, Passalacqua R, et al. (2014) Ramucirumab monotherapy for previously treated advanced gastric or gastro-oesophageal junction adenocarcinoma (REGARD): an international, randomised, multicentre, placebo controlled, phase 3 trial. Lancet 383: 31-39.

3. Han FH, Li HM, Zheng DH, He YL, Zhan WH (2010) The effect of the expression of vascular endothelial growth factor (VEGF)-C and VEGF receptor-3 on the clinical outcome in patients with gastric carcinoma. Eur J Surg Oncol 36: 1172-1179.

4. Choi JH, Oh YH, Park YW, Baik HK, Lee YY, et al. (2008) Correlation of vascular endothelial growth factor-D expression and VEGFR-3-positive vessel density with lymph node metastasis in gastric carcinoma. J Korean Med Sci 23: 592-597.

5. Dellinger MT, Meadows SM, Wynne K, Cleaver O, Brekken RA (2013) Vascular endothelial growth factor receptor-2 promotes the development of the lymphatic vasculature. PLoS One 8: e74686.

6. Chaudary N, Milosevic M, Hill RP (2011) Suppression of vascular endothelial growth factor receptor 3(VEGFR3) and vascular endothelial growth factor C(VEGFRC) inhibits hypoxia-induced lymph node metastases in cervix cancer. Gynecol Oncol 123: 393-400. 